\title{
Ständige Kommissionen Der Internationalen
} Radiologenkongresse

International Commission for Stage-grouping and for the Presentation of the Results I.C. P.

Prasident:

Prof. H. R. Schinz (Schweiz)

Prof. E. Berven (Schweden). Dr. J. Clemmesen (Danemark). Dr. P. F. Denoix (Frankreich). Prof. F. Perussia (Italien).

R.

Dr. F. E. Leborgne (Uruguay). Prof. H. F. J. Maisin (Belgien). Prof. R. McWhirter (GroBbritannien). Dr. K. P. Mody (Indien). Dr. V. V. Portmann (U.S.A.). Dr. E. Pendergrass (U.S.A.). Dr. A. II. Sellers (Canada).

International Commission on Radiological Protection I.C.R.P.

Prasident:

R. M. Sievert (Schweden

Vizeprasident: G. Failla (U.S.A.)

Sekretar:

W. Binks (GroBbritannien)

Mitglieder:

L. Bugnard (Frankreich)

H. Holthusen (Deutschland)

J. C. Jacobsen (Danemark)

R. G. Jaeger (Deutschland)

W. V. Mayneord (GroBbritannien)

K. Z. Morgan (U.S.A.)

R. S. Stone (U.S.A.)

L. S. Taylor (U.S.A.)

E. A. Watkinson (Canada)

International Commission on Radiological Units I.C.R.U.

Prasident:

L. S. Taylor (U.S.A.).

Vizeprasident:

L. H. Gray (GroBbritannien).

Sekretar:

H. 0. Wyckoff (U.S.A.).

Mitglieder:
A. Allisy (Frankreich).
R. H. Chamberlain (U.S.A.). 
F. Ellis (GroBbritannien).

G. Failla (U.S.A.).

H. Holthusen (Deutschland). H. E. Johns (Canada). F. E. Leborgne (Uruguay). E. J. Oosterkamp (Holland). B. Rajewsky (Deutschland). R. Sievert (Schweden).

* Laut Sehreiben vom 18. Februar 1957 von W. Bjnks hat diese Kommission Zusammensetzung. 